\title{
Maternal anemia is a potential risk factor for anemia in children aged 6-59 months in Southern Africa: a multilevel analysis
}

\author{
Peter A. M. Ntenda', Owen Nkoka', Paul Bass ${ }^{1,2}$ and Thomas Senghore ${ }^{1,2^{*}}$ (D)
}

\begin{abstract}
Background: The effect of maternal anemia on childhood hemoglobin status has received little attention. Thus, we examined the potential association between maternal anemia and childhood anemia (aged 6-59 months) from selected Southern Africa countries.

Methods: A cross-sectional study using nationally representative samples of children aged 6-59 months from the 2010 Malawi, 2011 Mozambique, 2013 Namibia, and 2010-11 Zimbabwe demographic and health surveys (DHS) was conducted. Generalized linear mixed models (GLMMs) were constructed to test the associations between maternal anemia and childhood anemia, controlling for individual and community sociodemographic covariates.

Results: The GLMMs showed that anemic mothers had increased odds of having an anemic child in all four countries; adjusted odds ratio ( $\mathrm{aOR}=1.69$ and $95 \%$ confidence interval [Cl]:1.37-2.13) in Malawi, (aOR=1.71; 95\% Cl: 1.37-2.13) in Mozambique, $(\mathrm{aOR}=1.55 ; 95 \% \mathrm{Cl}: 1.08-2.22)$ in Namibia, and (aOR $=1.52 ; 95 \% \mathrm{Cl}: 1.25-1.84)$ in Zimbabwe. Furthermore, the odds of having an anemic child was higher in communities with a low percentage of anemic mothers ( $\mathrm{aOR}=1.52$; 95\% Cl: 1.19-1.94) in Mozambique.

Conclusions: Despite the long-standing efforts to combat childhood anemia, the burden of this condition is still rampant and remains a significant problem in Southern Africa. Thus, public health strategies aimed at reducing childhood anemia should focus more on addressing infections, and micronutrient deficiencies both at individual and community levels in Southern Africa.
\end{abstract}

Keywords: Malawi, Mozambique, Namibia, Zimbabwe, Maternal anemia, Childhood anemia, Multilevel

\section{Background}

Anemia, defined as the volume of packed red blood cells/ hematocrit $(\mathrm{Hct})$ or hemoglobin $(\mathrm{Hb})$ concentration greater than two standard deviation (2SD) below mean for age, may be due to three general causes (i.e. blood loss, increased red blood cell $(\mathrm{RBC})$ destruction or reduced $\mathrm{RBC}$ production) $[1,2]$. The major physiologic impact of anemia is reduced oxygen delivery to tissues, resulting in both compensatory responses and acute or chronic consequences including poor growth, decreased activity, impaired cognitive performance, behavioral, motor development and limited cardiovascular reserve [1, 3-5].

\footnotetext{
* Correspondence: tsenghore@utg.edu.gm; tsenghore@gmail.com ${ }^{1}$ School of Public Health, College of Public Health, Taipei Medical University, No.250, Wu-Hsing St, Taipei City 110, Taiwan, R.O.C.

${ }^{2}$ School of Medicine and Allied Health Sciences, University of The Gambia, P.O. Box 1646, Independence Drive, Banjul, The Gambia
}

Anemia is a major public health problem in developing countries and a direct cause of childhood mortality and morbidity [6, 7]. Globally, 43\% of preschool-age children are anemic $[5,8,9]$ and $28.5 \%$ of these children reside in sub-Saharan Africa (SSA), which presents a startling prevalence rate of $67 \%$ [10]. The causes of anemia are multifactorial, however, about $50 \%$ of all anemia cases are due to iron deficiency $[11,12]$, although other factors such as micronutrient deficiencies (i.e., folate, riboflavin, and vitamins A and B12) [12-14], acute and chronic inflammation (i.e., malaria, tuberculosis, and HIV) $[13,15,16]$, and inherited or acquired disorders that affect $\mathrm{Hb}$ synthesis, $\mathrm{RBC}$ production, or RBC survival (i.e., hemoglobinopathies) $[15,17]$, can all be etiologies of anemia. In addition to nutritional and pathological factors, previous researchers have demonstrated other factors such

(c) The Author(s). 2018 Open Access This article is distributed under the terms of the Creative Commons Attribution 4.0 International License (http://creativecommons.org/licenses/by/4.0/), which permits unrestricted use, distribution, and reproduction in any medium, provided you give appropriate credit to the original author(s) and the source, provide a link to the Creative Commons license, and indicate if changes were made. The Creative Commons Public Domain Dedication waiver (http://creativecommons.org/publicdomain/zero/1.0/) applies to the data made available in this article, unless otherwise stated. 
as a child's characteristics [11, 18-20], maternal characteristics [18-20], household characteristics [19, 20], and community characteristics [20], also have impacts on childhood anemia.

The World Health Organization (WHO) estimated that over $50 \%$ of all women living in developing countries are anemic, compared with $18 \%$ in industrialized countries [21]. However, the burden of this condition is more pervasive in Asia and Africa where 60 and 52\% of women are estimated to be anemic respectively [21]. Previous studies have reported that maternal anemia during pregnancy is associated with a higher risk of low birth weight, preterm birth, perinatal and neonatal mortality, maternal morbidity and mortality, and low productivity [22-24]. Regardless, a large body of research has established links between maternal anemia in pregnancy and poor infant birth outcomes. However, little is known whether maternal anemia, in general, has an influence on childhood anemia after delivery. Thus, we aimed to investigate whether there is an association between maternal anemia and childhood anemia in four Southern African countries, controlling for a wide range of individual- and community-level sociodemographic factors.

\section{Methods}

\section{Study design, sampling technique, and data collection}

This study utilized demographic and health survey (DHS) data from four Southern African countries (2010 Malawi, 2011 Mozambique, 2013 Namibia, and Zimbabwe 2010-11). Methodologies used in these surveys have been previously described [25]. In brief, the surveys utilized a stratified two-stage cluster design. In the first stage, clusters were randomly selected from master sampling frames (enumeration area). The second stage selected a systematic sample of households from the communities (clusters). Information was collected from women aged 15-49 years, who had under-5 children prior to the survey. With the aid of an interviewer administered questionnaire information on sociodemographic, economic, environmental, immunization, anthropometric, household characteristics, child health care and population health indicators were collected. All the above surveys had response rates of more than $90 \%$. A random procedure was conducted to select one child per mother to avoid the clustering effects, which generated final sample sizes of 2507, 1933, 1116, and 2578 in Malawi, Mozambique, Namibia and Zimbabwe respectively. The HemoCue blood hemoglobin system (HemoCue 201+; Ängelholm, Sweden) [26] was utilized for $\mathrm{Hb}$ testing for children and mothers using finger prick blood. The HemoCue system is a rapid and widely used system in both clinical and survey settings that is comparable to standard laboratory techniques [27].

\section{Measures}

\section{Outcome variable}

Anemia in children under the age of 5 was the outcome variables in this study, defined as per WHO recommendations $(\mathrm{Hb}<11 \mathrm{~g} / \mathrm{dL})[1,3]$.

\section{Main independent variables}

The main predictor variable of this study was maternal anemia, used as both continuous ( $\mathrm{Hb}$ concentration) and categorical variable (cut-off of $<12 \mathrm{~g} / \mathrm{dL}$ as per WHO $[1,3])$.

\section{Covariates \\ Individual-level/maternal and household-level factors}

We selected covariates to adjust for possible confounding factors in the analyses (control variables). There were a total of twelve individual/household-level variables included in this study. Child-specific factors included child's sex (male or female), child's age (in months) (6$11,12-23,24-35,36-47$, and 48-59 months), birth order (1, 2, 3, 4 and above), history of fever (occurrence of fever in the last two weeks) diarrhea in the last two weeks (an episode of diarrhea, defined as passage of three or more loose or liquid stool in $24 \mathrm{~h}$ [28]), childhood stunting, and wasting (stunting was defined as moderate and severe - that is below minus two standard deviations $(<-2 \mathrm{SD})$ from median height-for-age $\mathrm{z}$-scores of reference population whilst underweight was defined as moderate and severe - that is below minus two standard deviations $(<-2 \mathrm{SD})$ from median weight-for-age z-score of reference population) [29]. Maternal and household characteristics include age in years $(15-24,25-34$, and $\geq 35$ years), educational attainment (no formal education, primary education, and secondary and higher education), and biofuel smoke exposure (dirty or clean fuel use). The use of electricity, natural gas, biogas, and kerosene for cooking were regarded as clean fuel whilst wood, straw, animal dung, and crop residues used as cooking fuels were defined as dirty fuel [30,31]. Parity was categorized into 1, 2, 3, 4, 5 and above children, and household wealth status was categorized into poorest, poor, middle, rich and richest. The wealth index was generated through a principal component analysis that utilized data on the household's ownership of selected assets. The household asset scores generated were then categorized into quintiles following standardization [32].

\section{Community-level factors}

We included six variables. One variable indicated an area of residence (i.e., urban or rural). Six continuous variables assessed community maternal anemia, community total children ever born, community wealth, community female education, community distance to a health facility and community water supply. The primary 
sample unit (PSU) in the DHS data was used to define a community. Individual-level data were aggregated to create continuous community-level factors [33]. The community wealth was defined as a percentage of households categorized as $60 \%$ and above of wealth index, similarly community female education was defined as the percentage of women with primary school education and above. Community maternal anemia was defined as the percentage of women with $\mathrm{Hb}$ levels less than $12 \mathrm{~g} / \mathrm{dL}$. Community parity was the percent of women with a fertility rate of 5 children and above and community distance to the health facility was defined as a percentage of household who perceived the distance to the nearest health facility as a big problem. Community water supply was defined as the percent of household with access to clean and safe drinking water sources specified by WHO/UNICEF [34]. All community-level variables were categorized as "low", "medium" and "high" depending upon each variable's tertiles.

\section{Statistical analyses}

SAS software version 9.4 (SAS Institute Inc., Cary, NC, USA) was used for all statistical analysis. Due to the possible effect of clustering, data on baseline sociodemographics were weighted. Frequencies and percentages were reported for categorical variables. The differences in maternal anemia, sociodemographic, nutrition, morbidity outcomes, and community factors according to anemia status were compared using chi-square tests. Cochran-Armitage Trend Test was used to assess trends. A two-level multilevel multivariable logistic regression (MMLR) analysis with a logit-link function and binomial distribution was applied, fitting four different generalized linear mixed models. Since children from the same community may present similar characteristics than individuals from different communities, we adjusted for the correlated individual responses nested under a single community using multilevel models. To avoid large type II errors, only variables significant at $p \leq 0.25$ in the bivariate analysis were included in this analysis [35]. Four models were constructed in this analysis. Model 1 was a null model for assessing the total variance between communities. Model 2 contained maternal anemia and individual-level factors, and model 3 contained only community-level factors. Model 4 included maternal anemia status, individual- and community-level factors. Only the final model is presented in this paper. Results of the multivariable analyses are reported in terms of adjusted odds ratios (aORs) with their $p$-values and 95\% confidence intervals (CIs). Intra-class correlations (ICCs) and percentage change in variation (PCV) were reported to assess the extent to which community variances were explained in each model. Model fits were assessed using a deviation information criterion (DIC). Prior to multivariate analysis, multicollinearity was examined using variance inflation factor (VIF) (Additional file 1). Two variables (Child's birth order and Total children ever born) had high VIF values. Figure 1 shows the fit diagnostics for childhood anemia, which showed that a lot of variation in the model was unexplained (proportion less).

\section{Ethics statement}

The study protocols were reviewed and approved by the Malawi Health Science Research Committee, National Bioethics Commission of Mozambique (Comissao de Bioetica Nacional), Namibia Ministry of Health and Social Services, Medical Research Council of Zimbabwe and the Institutional Review Board (IRB) of ICF Macro, and the Centers for Disease Control (CDC) in Atlanta. Prior to the interview and blood sample collection, all participants provided written informed consent. Permission was granted by the DHS program for the use of the data.

\section{Results \\ Sample characteristics}

The prevalence of childhood anemia was 63.8, 70.0, 49.4, and 58.6\% in Malawi, Mozambique, Namibia, and Zimbabwe respectively. At the individual- level, maternal anemia was estimated at 26.7, 53.4, 19.8 and $25.1 \%$ with community-level at 20.7, 58.6, 15.8 and $24.3 \%$ in Malawi, Mozambique, Namibia, and Zimbabwe respectively (Table 1 ).

Table 2 presents results of the bivariate analysis for all four countries. The prevalence of childhood anemia was found to be significantly higher among children whose mothers were anemic, children who were exposed to biofuel smoke (Mozambique), males (Namibia), children aged 6-23 months, children with a recent history of fever (Malawi, Mozambique and Namibia), diarrhea and chronic undernutrition (Malawi, Mozambique and Zimbabwe). Furthermore, childhood anemia was also significantly higher in children whose mothers were aged 15-24 years (Malawi, Namibia and Zimbabwe), children born to mothers with no formal education (Malawi and Mozambique) and in children resident in poor and poorest households (Malawi, Mozambique and Namibia). Additionally, the prevalence was also significantly higher in children residing in rural areas (Malawi and Mozambique), communities with a higher percentage of anemic women, low parity (Malawi and Mozambique) low wealth (Malawi, Mozambique and Namibia), low female education (Malawi and Mozambique), high perceived distance to the health facility as a big problem (Malawi and Mozambique), and low access to safe water (Malawi and Mozambique). 


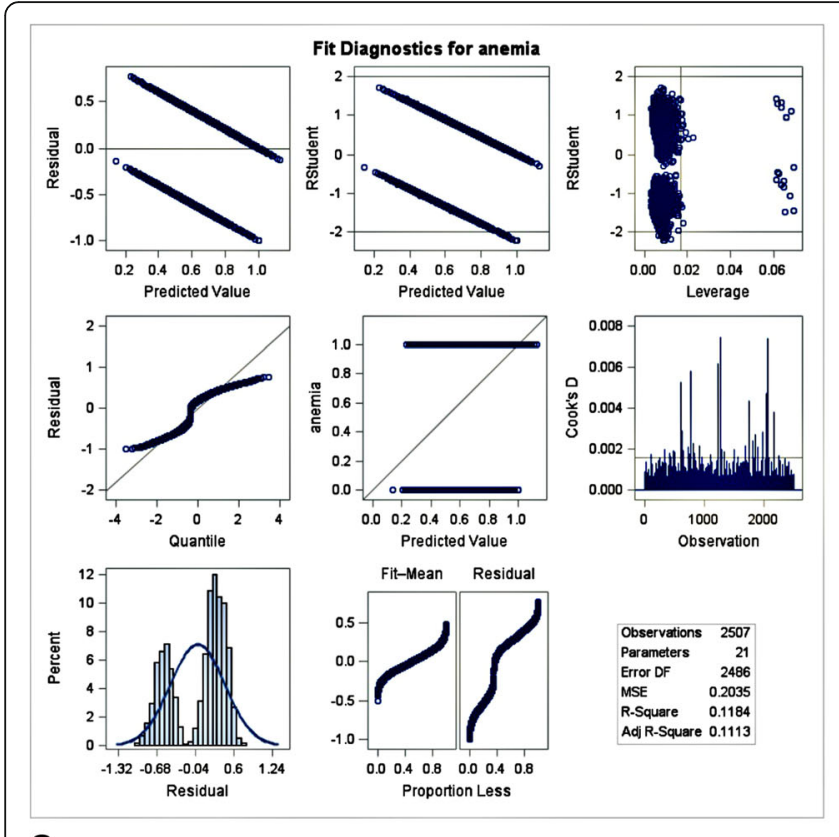

a

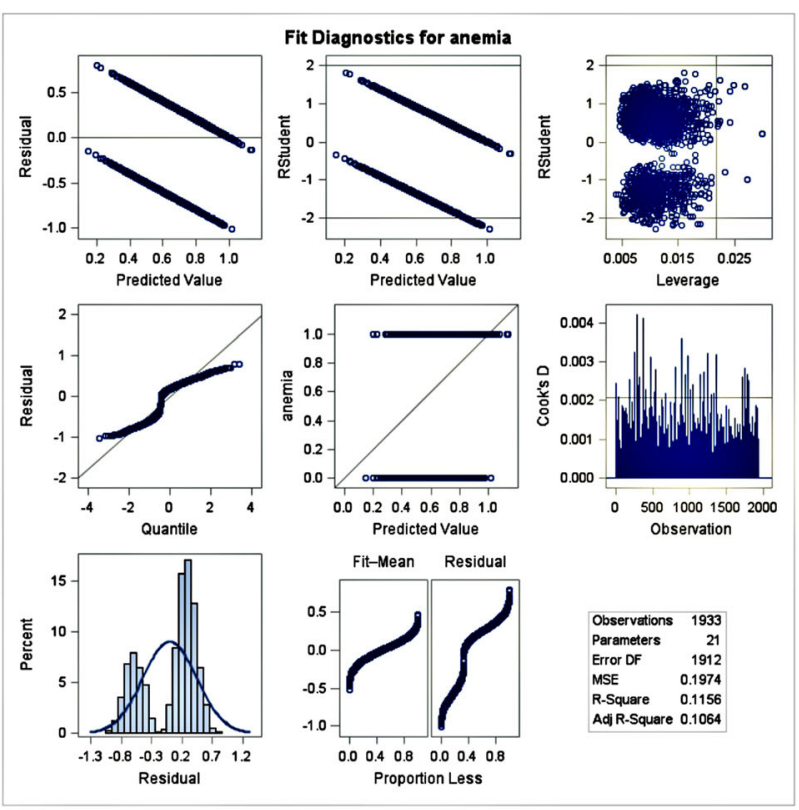

b
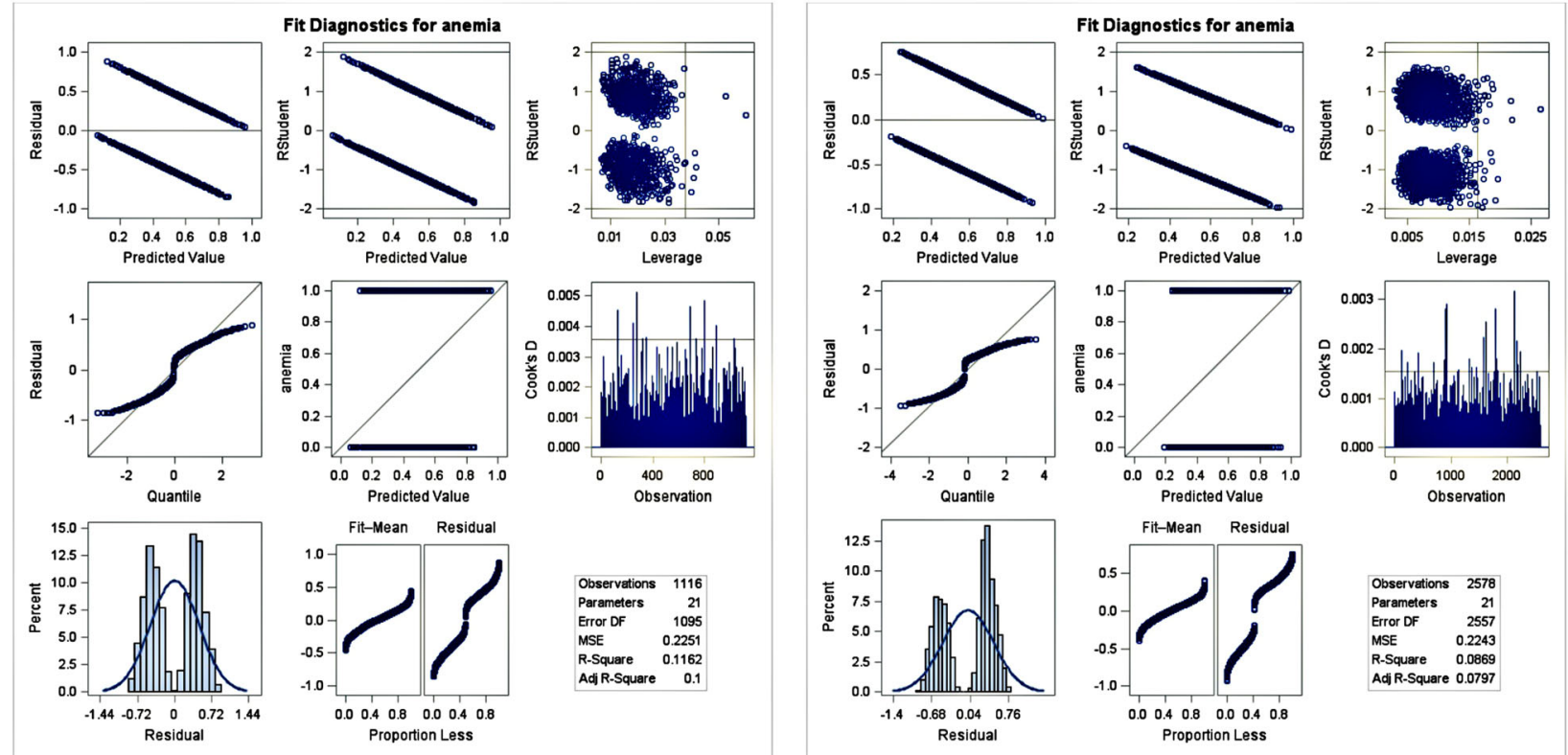

\begin{tabular}{ll} 
Error DF & 1095 \\
\hline MSE & 0.2251
\end{tabular}

$\begin{array}{ll}\text { MSE } & 0.2251 \\ \text { R-Square } & 0.1162\end{array}$

R-Square 0.1162

C
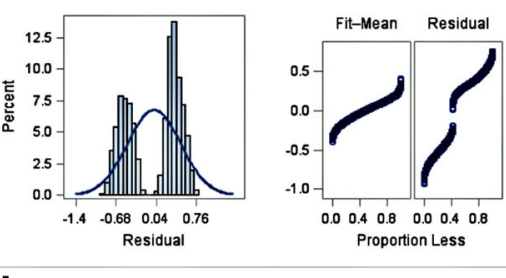

Observations 2578

$\begin{array}{lr}\text { Parameters } & 21 \\ \text { Error } & 2557\end{array}$

\begin{tabular}{lr} 
Error DF & 2557 \\
MSE & 0.2243 \\
\hline RS & 0.069
\end{tabular}

$\begin{array}{lr}\text { R-Square } & 0.0869 \\ \text { Adj R-Square } & 0.0797\end{array}$

d

Fig. 1 Fit Diagnostics for Childhood Anemia in (a) Malawi, (b) Mozambique, (c) Namibia, and (d) Zimbabwe

Effect of maternal anemia and other covariates on

\section{childhood anemia}

Table 3 showed the results of multivariate multilevel logistic regression analyses (Model 4 only). Compared to non-anemic mother, anemic mothers were $(\mathrm{aOR}=1.69$; 95\% CI: $1.37-2.13), 29 \% \quad(\mathrm{aOR}=1.71 ; 95 \% \mathrm{CI}: 1.37-$ $2.13),(\mathrm{aOR}=1.55 ; 95 \% \mathrm{CI}: 1.08-2.22)$ and $(\mathrm{aOR}=1.52$; 95\% CI: 1.25-1.84) significantly more likely to have anemic children in Malawi, Mozambique, Namibia, and
Zimbabwe respectively. Additionally, compared to communities with a high percentage of anemic women, communities with a low percentage of anemic women were $(\mathrm{aOR}=1.52 ; 95 \% \mathrm{CI}: 1.19-1.94)$ more likely to have anemic children in Mozambique.

For individual-level factors, across all countries, the odds of childhood anemia was decreased with increased age of children. In Namibia female children were significantly less likely to be anemic compared to their male 
Table 1 Sample characteristics of childhood anemia for the four selected Southern African countries

\begin{tabular}{|c|c|c|c|c|}
\hline \multirow[t]{3}{*}{ Characteristic } & Malawi & Mozambique & Namibia & Zimbabwe \\
\hline & $N=2507$ & $N=1933$ & $N=1116$ & $N=2578$ \\
\hline & $n^{*}(\%)^{* *}$ & $n^{*}(\%)^{* *}$ & $n^{*}(\%)^{* *}$ & $n^{*}(\%)^{* *}$ \\
\hline Childhood anemia (\%) & 63.8 & 70.0 & 49.4 & 58.6 \\
\hline \multicolumn{5}{|l|}{ Exposure variable } \\
\hline \multicolumn{5}{|c|}{ Maternal anemic status } \\
\hline Nonanemic & $1850(73.3)$ & $934(46.6)$ & $908(80.2)$ & $1898(74.9)$ \\
\hline Anemic & $657(26.7)$ & $999(53.4)$ & $208(19.8)$ & $680(25.1)$ \\
\hline \multicolumn{5}{|l|}{ Individual-level } \\
\hline \multicolumn{5}{|c|}{ Biofuel smoke exposure ${ }^{\beta}$} \\
\hline No & $17(0.8)$ & $91(2.9)$ & $352(34.3)$ & $552(21.0)$ \\
\hline Yes & $2490(99.2)$ & $1842(97.1)$ & $764(65.7)$ & $2026(79.0)$ \\
\hline \multicolumn{5}{|l|}{ Sex of child } \\
\hline Male & $1253(48.9)$ & $929(48.5)$ & $533(47.3)$ & $1299(49.9)$ \\
\hline Female & $1254(51.1)$ & $1004(51.5)$ & $583(52.7)$ & $1279(50.1)$ \\
\hline \multicolumn{5}{|l|}{ Child age in months } \\
\hline $6-11$ & $268(11.4)$ & $247(13.2)$ & $153(14.3)$ & $394(14.8)$ \\
\hline $12-23$ & $616(25.1)$ & $502(26.0)$ & $294(25.1)$ & $628(23.9)$ \\
\hline $24-35$ & $579(22.2)$ & $459(23.3)$ & $253(22.4)$ & $627(24.8)$ \\
\hline $36-47$ & $586(23.0)$ & 377 (19.9) & $234(21.0)$ & $509(20.5)$ \\
\hline $48-59$ & $458(18.3)$ & $348(17.6)$ & $182(17.2)$ & $420(16.0)$ \\
\hline \multicolumn{5}{|l|}{ Child's birth order } \\
\hline 1 & $498(20.8)$ & $472(23.8)$ & $351(32.6)$ & $832(32.1)$ \\
\hline 2 & $437(17.8)$ & $347(17.3)$ & $278(25.2)$ & $680(26.1)$ \\
\hline 3 & $405(16.2)$ & $284(14.2)$ & $177(16.1)$ & $449(17.9)$ \\
\hline $4+$ & $1167(45.2)$ & $830(44.7)$ & $310(26.1)$ & $617(23.9)$ \\
\hline \multicolumn{5}{|l|}{ Recent episodes of fever } \\
\hline No & $1590(62.5)$ & $1658(84.7)$ & $310(70.5)$ & $2285(88.4)$ \\
\hline Yes & $917(37.5)$ & $275(15.3)$ & $329(29.5)$ & $293(11.6)$ \\
\hline \multicolumn{5}{|c|}{ Recent episodes of diarrhea } \\
\hline No & $2084(83.5)$ & 1709 (87.3) & $868(76.5)$ & $2158(83.0)$ \\
\hline Yes & $423(16.5)$ & $224(12.7)$ & $248(23.5)$ & $420(17.0)$ \\
\hline \multicolumn{5}{|l|}{ Children are stunted } \\
\hline No & $1303(51.2)$ & $1062(50.7)$ & $855(77.2)$ & $1690(65.5)$ \\
\hline Yes & $1204(48.8)$ & $871(49.3)$ & $261(22.8)$ & $888(34.5)$ \\
\hline \multicolumn{5}{|c|}{ Children are underweight } \\
\hline No & $2179(86.8)$ & $1657(84.0)$ & $976(87.2)$ & $2304(89.9)$ \\
\hline Yes & $328(13.2)$ & $276(16.0)$ & $140(12.8)$ & $274(10.1)$ \\
\hline \multicolumn{5}{|l|}{ Mother's age in years } \\
\hline $15-24$ & $773(31.1)$ & $630(32.8)$ & $337(28.0)$ & $870(33.8)$ \\
\hline $25-34$ & $1182(46.6)$ & $817(41.0)$ & $477(44.7)$ & $1227(46.9)$ \\
\hline $35-49$ & $552(22.3)$ & $486(26.2)$ & $302(27.3)$ & $481(19.3)$ \\
\hline \multicolumn{5}{|c|}{ Mother's educational level } \\
\hline No education & $423(17.6)$ & $740(40.2)$ & $86(5.9)$ & $60(2.3)$ \\
\hline Primary & 1778 (70.5) & $993(52.4)$ & $261(22.8)$ & 848 (32.1) \\
\hline
\end{tabular}


Table 1 Sample characteristics of childhood anemia for the four selected Southern African countries (Continued)

\begin{tabular}{|c|c|c|c|c|}
\hline \multirow[t]{3}{*}{ Characteristic } & Malawi & Mozambique & Namibia & Zimbabwe \\
\hline & $N=2507$ & $N=1933$ & $N=1116$ & $N=2578$ \\
\hline & $n^{*}(\%)^{* *}$ & $n^{*}(\%)^{* *}$ & $n^{*}(\%)^{* *}$ & $n^{*}(\%)^{* *}$ \\
\hline Secondary and above & $306(11.9)$ & $200(7.4)$ & $769(71.3)$ & $1670(65.6)$ \\
\hline \multicolumn{5}{|l|}{ Total children ever born } \\
\hline 1 & $361(15.0)$ & $374(19.1)$ & $312(29.2)$ & $703(27.0)$ \\
\hline 2 & $451(18.6)$ & $348(17.5)$ & $286(26.1)$ & $723(28.0)$ \\
\hline 3 & $426(17.6)$ & $305(14.8)$ & $190(17.0)$ & $486(19.4)$ \\
\hline 4 & $403(14.6)$ & $262(13.8)$ & $115(9.7)$ & $308(11.7)$ \\
\hline $5+$ & $866(34.2)$ & $644(34.8)$ & $213(18.0)$ & $358(13.9)$ \\
\hline \multicolumn{5}{|l|}{ Household wealth index } \\
\hline Poorest & $484(18.7)$ & $431(28.0)$ & $247(23.7)$ & $676(24.4)$ \\
\hline Poor & $623(25.8)$ & $445(25.3)$ & $244(21.7)$ & $562(22.3)$ \\
\hline Middle & $610(23.9)$ & $406(20.5)$ & $235(19.2)$ & $501(20.4)$ \\
\hline Richer & 509 (19.3) & 415 (18.5) & $248(21.5)$ & $514(20.2)$ \\
\hline Richest & $281(12.5)$ & $236(7.7)$ & $142(13.9)$ & $325(12.7)$ \\
\hline
\end{tabular}

Community-level (clusters)

Place of residence

\begin{tabular}{|c|c|c|c|c|}
\hline Urban & $159(8.2)$ & $407(17.8)$ & $480(44.5)$ & $633(25.1)$ \\
\hline Rural & $2348(91.8)$ & $1526(82.2)$ & $636(55.5)$ & $1945(74.9)$ \\
\hline \multicolumn{5}{|c|}{ Community maternal anemia ${ }^{\dagger}$} \\
\hline Low anemia & $2020(79.3)$ & $846(41.4)$ & $967(884.2)$ & $2010(75.7)$ \\
\hline High anemia & $487(20.7)$ & 1087 (58.6) & $149(15.8)$ & $568(24.3)$ \\
\hline \multicolumn{5}{|c|}{ Community parity ${ }^{\jmath}$} \\
\hline Low & $913(38.7)$ & $711(30.8)$ & $860(79.1)$ & $1171(45.3)$ \\
\hline High & $1594(61.3)$ & $1222(69.2)$ & $256(20.9)$ & $1407(52.7)$ \\
\hline \multicolumn{5}{|c|}{ Community wealth ${ }^{\dagger \dagger}$} \\
\hline Low & $2041(79.7)$ & $1328(76.7)$ & $794(69.8)$ & $1753(67.8)$ \\
\hline High & $466(20.3)$ & $605(23.3)$ & $322(30.1)$ & $825(32.2)$ \\
\hline \multicolumn{5}{|c|}{ Community female education ${ }^{\S}$} \\
\hline Low & $585(24.4)$ & $1230(66.5)$ & $127(7.8)$ & $594(22.8)$ \\
\hline High & $1922(75.6)$ & $703(33.5)$ & $989(92.2)$ & $1984(77.2)$ \\
\hline \multicolumn{5}{|c|}{ Community distance to $\mathrm{HF}^{¥}$} \\
\hline Low & $686(30.1)$ & $672(28.7)$ & $682(62.9)$ & $1031(41.0)$ \\
\hline High & $1821(69.9)$ & $1261(71.3)$ & $434(37.1)$ & $1547(59.0)$ \\
\hline \multicolumn{5}{|c|}{ Community safe water access $^{\ddagger}$} \\
\hline Low & $619(26.3)$ & $1336(75.6)$ & $434(19.4)$ & $497(19.3)$ \\
\hline High & $1888(73.7)$ & $597(24.4)$ & 922 (80.6) & 2081 (80.7) \\
\hline
\end{tabular}

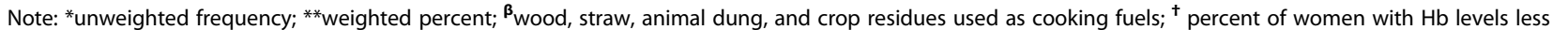
than $12 \mathrm{~g} / \mathrm{dL}_{\text {; }}$ ' percent of women with fertility rate of 5 children and above; ${ }^{{ }^{+\dagger}}$ percent of households categorized above $60 \%$ of wealth index; ${ }^{\S}$ percent of women with primary school education and above; ${ }^{*}$ percent of households perceived distance to the nearest health facility as a big problem; ${ }^{*}$ percent of household with access to clean and safe drinking water sources specified by WHO/Unicef [34]; HF, health facility

counterparts $(\mathrm{OR}=0.76$; 95\% CI: 0.59-0.99). Compared to children with no history of fever in the last two weeks, children with fever were $(\mathrm{aOR}=1.57 ; 95 \% \mathrm{CI}$ : $1.29-1.90$ and $\mathrm{aOR}=1.68$; 95\% CI: $1.19-2.37$ ) more likely to be anemic in Malawi and Mozambique respectively. Children with no history of stunting were 19\% $(\mathrm{aOR}=0.81 ; 95 \%$ CI: $0.67-0.98)$ and $33 \%(\mathrm{aOR}=0.67 ; 95 \%$ CI: $0.52-0.85)$ less likely to be anemic in Malawi and Mozambique respectively. The richest households were $(\mathrm{aOR}=0.69 ; 95 \% \mathrm{Cl}=0.48-0.99)$ less likely to have anemic 
Table 2 Bivariate analysis of childhood anemia for the four selected Southern African countries

\begin{tabular}{|c|c|c|c|c|c|c|c|c|}
\hline \multirow[t]{3}{*}{ Characteristic } & \multicolumn{2}{|c|}{ Malawi } & \multicolumn{2}{|c|}{ Mozambique } & \multicolumn{2}{|c|}{ Namibia } & \multicolumn{2}{|c|}{ Zimbabwe } \\
\hline & \multicolumn{2}{|c|}{ Anemia* } & \multicolumn{2}{|c|}{ Anemia* } & \multicolumn{2}{|c|}{ Anemia* } & \multicolumn{2}{|c|}{ Anemia* } \\
\hline & No $\%$ & Yes \% & No $\%$ & Yes \% & No $\%$ & Yes $\%$ & No $\%$ & Yes $\%$ \\
\hline \multicolumn{9}{|l|}{ Exposure variable } \\
\hline \multicolumn{9}{|l|}{ Maternal anemic status } \\
\hline No anemic & 38.76 & $61.24^{\mathrm{a}}$ & 40.04 & $59.96^{a}$ & 50.88 & $49.12^{\mathbf{b}}$ & 44.26 & $55.74^{\mathrm{a}}$ \\
\hline Anemic & 26.18 & 73.82 & 26.23 & 73.77 & 39.42 & 60.58 & 35.74 & 64.26 \\
\hline \multicolumn{9}{|l|}{ Individual-level } \\
\hline \multicolumn{9}{|l|}{ Biofuel smoke exposure ${ }^{\beta}$} \\
\hline No & 58.82 & 41.18 & 53.85 & $46.15^{\mathbf{a}}$ & 52.56 & 47.44 & 43.12 & 56.88 \\
\hline Yes & 35.30 & 64.70 & 31.87 & 68.13 & 46.99 & 53.01 & 41.71 & 58.29 \\
\hline \multicolumn{9}{|l|}{ Sex of child } \\
\hline Male & 34.24 & 65.76 & 32.72 & 67.28 & 45.22 & $54.78^{c}$ & 41.57 & 58.43 \\
\hline Female & 36.68 & 63.32 & 33.07 & 66.93 & 51.97 & 48.03 & 42.46 & 57.54 \\
\hline \multicolumn{9}{|l|}{ Child age in months } \\
\hline $6-11$ & 9.33 & $90.67^{\mathrm{a}}$ & 19.43 & $80.57^{\mathrm{a}}$ & 33.99 & $66.01^{a}$ & 28.17 & $71.83^{\mathrm{a}}$ \\
\hline $12-23$ & 25.65 & 74.35 & 25.70 & 74.30 & 31.97 & 68.03 & 27.07 & 72.93 \\
\hline $24-35$ & 35.75 & 64.25 & 33.55 & 66.45 & 49.80 & 50.20 & 43.06 & 56.94 \\
\hline $36-47$ & 45.39 & 54.61 & 37.67 & 62.33 & 62.82 & 37.18 & 54.03 & 45.97 \\
\hline $48-59$ & 50.87 & 49.13 & 46.84 & 53.16 & 68.68 & 31.32 & 61.19 & 38.81 \\
\hline \multicolumn{9}{|l|}{ Child's birth order } \\
\hline 1 & 31.93 & 68.07 & 32.84 & 67.16 & 44.73 & 55.27 & 41.11 & 58.89 \\
\hline 2 & 38.52 & 62.01 & 35.45 & 64.55 & 50.36 & 49.64 & 40.88 & 59.12 \\
\hline 3 & 38.52 & 61.48 & 31.69 & 68.31 & 51.41 & 48.59 & 45.66 & 54.34 \\
\hline $4+$ & 34.96 & 65.04 & 32.29 & 67.71 & 50.32 & 49.68 & 41.82 & 58.18 \\
\hline \multicolumn{9}{|l|}{ Recent episodes of fever } \\
\hline No & 39.94 & $60.06^{a}$ & 34.74 & $65.26^{\mathrm{a}}$ & 51.59 & $48.41^{\mathbf{b}}$ & 42.36 & 57.64 \\
\hline Yes & 27.70 & 72.30 & 21.82 & 78.18 & 41.95 & 58.05 & 39.25 & 60.75 \\
\hline \multicolumn{9}{|c|}{ Recent episodes of diarrhea } \\
\hline No & 37.38 & $62.62^{a}$ & 33.94 & $66.06^{\mathbf{b}}$ & 52.19 & $47.81^{a}$ & 43.28 & $56.72^{\mathbf{b}}$ \\
\hline Yes & 26.00 & 74.00 & 25.00 & 75.00 & 36.69 & 63.31 & 35.48 & 64.52 \\
\hline \multicolumn{9}{|l|}{ Children are stunted } \\
\hline No & 38.60 & $61.40^{\mathrm{a}}$ & 38.14 & $61.86^{\mathbf{a}}$ & 50.06 & 49.94 & 43.55 & $56.45^{c}$ \\
\hline Yes & 32.06 & 67.94 & 26.52 & 73.48 & 44.44 & 55.56 & 39.08 & 60.92 \\
\hline \multicolumn{9}{|l|}{ Children are underweight } \\
\hline No & 36.71 & $63.29^{a}$ & 34.58 & $65.42^{\mathrm{a}}$ & 48.16 & 51.84 & 42.58 & 57.42 \\
\hline Yes & 27.13 & 72.87 & 22.83 & 77.17 & 52.86 & 47.14 & 37.23 & 62.77 \\
\hline \multicolumn{9}{|l|}{ Mother's age in years } \\
\hline $15-24$ & 31.44 & $68.56^{c}$ & 29.37 & 70.63 & 38.58 & $61.42^{\mathrm{a}}$ & 36.78 & $63.22^{\mathrm{a}}$ \\
\hline $25-34$ & 37.99 & 62.01 & 34.88 & 65.12 & 51.57 & 48.43 & 43.77 & 56.23 \\
\hline $35-49$ & 35.69 & 64.31 & 34.16 & 65.84 & 55.63 & 44.37 & 46.99 & 53.01 \\
\hline \multicolumn{9}{|l|}{ Mother's educational level } \\
\hline No education & 29.79 & $70.21^{\mathbf{b}}$ & 30.27 & $69.73^{a}$ & 47.67 & 52.33 & 38.33 & 61.67 \\
\hline Primary & 35.55 & 64.45 & 31.32 & 68.68 & 47.89 & 52.11 & 39.74 & 60.26 \\
\hline Secondary and above & 42.81 & 57.19 & 50.50 & 49.50 & 49.15 & 50.85 & 43.29 & 56.71 \\
\hline
\end{tabular}


Table 2 Bivariate analysis of childhood anemia for the four selected Southern African countries (Continued)

\begin{tabular}{|c|c|c|c|c|c|c|c|c|}
\hline \multirow[t]{3}{*}{ Characteristic } & \multicolumn{2}{|c|}{ Malawi } & \multirow{2}{*}{\multicolumn{2}{|c|}{$\frac{\text { Mozambique }}{\text { Anemia* }}$}} & \multirow{2}{*}{\multicolumn{2}{|c|}{$\frac{\text { Namibia }}{\text { Anemia* }}$}} & \multirow{2}{*}{\multicolumn{2}{|c|}{$\frac{\text { Zimbabwe }}{\text { Anemia* }}$}} \\
\hline & \multicolumn{2}{|c|}{ Anemia* } & & & & & & \\
\hline & No $\%$ & Yes $\%$ & No $\%$ & Yes \% & No $\%$ & Yes $\%$ & No $\%$ & Yes $\%$ \\
\hline \multicolumn{9}{|c|}{ Total children ever born } \\
\hline 1 & 26.87 & $73.13^{c}$ & 31.28 & 68.72 & 42.63 & $57.37^{c}$ & 37.41 & $62.59^{c}$ \\
\hline 2 & 37.92 & 62.08 & 36.49 & 63.51 & 50.70 & 49.30 & 43.43 & 56.57 \\
\hline 3 & 40.14 & 59.86 & 30.16 & 69.84 & 52.11 & 47.89 & 45.06 & 54.94 \\
\hline 4 & 33.75 & 66.25 & 35.11 & 64.89 & 49.57 & 50.43 & 40.26 & 59.74 \\
\hline $5+$ & 36.26 & 63.74 & 32.30 & 67.70 & 51.64 & 48.36 & 45.53 & 54.47 \\
\hline \multicolumn{9}{|c|}{ Household wealth index } \\
\hline Poorest & 28.93 & $71.07^{\mathrm{a}}$ & 24.13 & $75.87^{\mathbf{a}}$ & 50.20 & $49.80^{c}$ & 41.57 & 58.43 \\
\hline Poor & 33.71 & 66.29 & 28.54 & 71.46 & 42.21 & 57.79 & 40.57 & 59.43 \\
\hline Middle & 33.28 & 66.72 & 33.00 & 67.00 & 46.81 & 53.19 & 43.51 & 56.49 \\
\hline Richer & 39.69 & 60.31 & 36.63 & 63.37 & 48.79 & 51.21 & 40.08 & 59.92 \\
\hline Richest & 47.69 & 52.31 & 50.42 & 49.58 & 60.56 & 39.44 & 46.15 & 53.85 \\
\hline \multicolumn{9}{|c|}{ Community-level (clusters) } \\
\hline \multicolumn{9}{|c|}{ Place of residence } \\
\hline Urban & 45.91 & $54.09^{\mathbf{b}}$ & 43.00 & $57.00^{\mathbf{a}}$ & 50.00 & 50.00 & 40.28 & 59.72 \\
\hline Rural & 34.75 & 65.25 & 30.21 & 69.79 & 47.80 & 52.20 & 42.57 & 57.43 \\
\hline \multicolumn{9}{|c|}{ Community maternal anemia $^{\dagger}$} \\
\hline Low anemia & 37.23 & $62.77^{\mathbf{b}}$ & 39.36 & $60.64^{a}$ & 50.36 & $49.64^{\mathbf{b}}$ & 40.75 & $59.25^{c}$ \\
\hline High anemia & 28.13 & 71.87 & 27.87 & 72.13 & 38.26 & 61.74 & 46.48 & 53.52 \\
\hline \multicolumn{9}{|c|}{ Community parity } \\
\hline Low & 41.95 & $58.05^{\mathrm{a}}$ & 36.85 & $63.15^{\mathbf{b}}$ & 49.19 & 50.81 & 41.59 & 58.41 \\
\hline High & 31.74 & 68.26 & 30.61 & 69.39 & 47.27 & 52.73 & 42.36 & 57.64 \\
\hline \multicolumn{9}{|c|}{ Community wealth $^{\dagger+}$} \\
\hline Low & 33.56 & $66.44^{a}$ & 28.31 & $71.69^{a}$ & 46.47 & $53.53^{c}$ & 42.33 & 57.67 \\
\hline High & 43.78 & 56.22 & 42.98 & 57.02 & 54.35 & 45.65 & 41.33 & 58.67 \\
\hline \multicolumn{9}{|c|}{ Community female education ${ }^{\S}$} \\
\hline Low & 29.40 & $70.60^{a}$ & 29.35 & $70.65^{\mathbf{a}}$ & 44.09 & 55.91 & 41.25 & 58.75 \\
\hline High & 37.30 & 62.70 & 39.12 & 60.88 & 49.34 & 50.66 & 42.24 & 57.76 \\
\hline \multicolumn{9}{|c|}{ Community distance to $\mathrm{HF}^{¥}$} \\
\hline Low & 43.00 & $57.00^{\mathrm{a}}$ & 39.73 & $60.27^{a}$ & 50.15 & 49.85 & 41.61 & 58.39 \\
\hline High & 32.62 & 67.38 & 29.26 & 70.74 & 46.54 & 53.46 & 42.28 & 57.72 \\
\hline \multicolumn{9}{|c|}{ Community safe water access $^{\ddagger}$} \\
\hline Low & 31.66 & $68.34^{c}$ & 28.44 & $71.56^{\mathbf{a}}$ & 50.52 & 49.48 & 40.24 & 59.76 \\
\hline High & 36.71 & 63.29 & 42.88 & 57.12 & 48.37 & 51.63 & 42.43 & 57.57 \\
\hline
\end{tabular}

Note: ${ }^{\mathbf{a}} \boldsymbol{P}<0.0001 ;{ }^{\mathbf{b}} \boldsymbol{P}<0.001 ;{ }^{\mathbf{c}} \boldsymbol{P}<0.05 ; \quad$ Cochran-Armitage Trend Test - statistical trend analysis; ${ }^{*}$ Children with hemoglobin levels of less than $11 \mathrm{~g} / \mathrm{dL}$ adjusted for altitude; ${ }^{\boldsymbol{\beta}}$ wood, straw, animal dung, and crop residues used as cooking fuels ${ }^{\dagger}$ percent of women with $\mathrm{Hb}$ levels less than $12 \mathrm{~g} / \mathrm{dL} ;{ }^{\top}$ percent of women with fertility rate of 5 children and above; ${ }^{++}$percent of households categorized above $60 \%$ of wealth index; ${ }^{\S}$ percent of women with primary school education and above; ${ }^{*}$ percent of households perceived distance to the nearest health facility as a big problem; ${ }^{\ddagger}$ percent of household with access to clean and safe drinking water sources specified by WHO/Unicef [34]

children compared to the poorest households in Malawi. For community-level factors, children in communities with a high percentage of mothers perceiving distance to a health facility as a big problem were $(\mathrm{aOR}=1.33$; $95 \% \mathrm{CI}$ : 1.07-1.65) more likely to be anemic in Malawi.
The measure of variation (Model 4 only) shows that about $2,8,2$, and $2 \%$ of the community-level variance of childhood anemia were unexplained in Malawi, Mozambique, Namibia, and Zimbabwe respectively. On the other hand, the PCVs shows that $71,33,55$, and $26 \%$ 
Table 3 Measures of association and variation between individual- and community-level factors and childhood anemia

\begin{tabular}{|c|c|c|c|c|}
\hline Characteristic & Malawi & Mozambique & Namibia & Zimbabwe \\
\hline & aOR $95 \%(\mathrm{Cl}) / p$ & aOR 95\% (CI)/p & aOR 95\% (Cl)/p & aOR 95\% (Cl)/p \\
\hline \multicolumn{5}{|l|}{ Exposure variable } \\
\hline \multicolumn{5}{|l|}{ Maternal anemic status } \\
\hline Nonanemic & 1.00 & 1.00 & 1.00 & 1.00 \\
\hline Anemic & $1.69(1.35-2.12)^{\mathbf{a}}$ & $1.71(1.37-2.13)^{\mathbf{a}}$ & $1.55(1.08-2.22)^{\mathbf{c}}$ & $1.52(1.25-1.84)^{\mathbf{a}}$ \\
\hline Maternal Hb concentration & $0.64(0.55-0.55)^{\mathbf{a}}$ & $0.67(0.58-0.78)^{\mathbf{a}}$ & $0.70(0.54-0.91)^{\mathbf{b}}$ & $0.75(0.65-0.86)^{\mathbf{a}}$ \\
\hline \multicolumn{5}{|l|}{ Individual-level } \\
\hline \multicolumn{5}{|l|}{ Biofuel smoke exposure ${ }^{\beta}$} \\
\hline No & NA & 1.00 & 1.00 & NA \\
\hline Yes & NA & $0.93(0.50-1.73)$ & $0.92(0.58-1.45)$ & NA \\
\hline \multicolumn{5}{|l|}{ Sex of child } \\
\hline Male & 1.00 & NA & 1.00 & NA \\
\hline Female & $0.93(0.78-1.11)$ & NA & $0.76(0.59-0.99)^{a}$ & NA \\
\hline \multicolumn{5}{|l|}{ Child age in months } \\
\hline $6-11$ & 1.00 & 1.00 & 1.00 & 1.00 \\
\hline $12-23$ & $0.28(0.17-0.44)^{\mathbf{a}}$ & $0.58(0.39-0.88)^{\mathbf{a}}$ & $1.08(0.70-1.69)$ & $0.99(0.74-1.33)$ \\
\hline $24-35$ & $0.17(0.11-0.27)^{\mathbf{a}}$ & $0.36(0.24-0.54)^{a}$ & $0.51(0.33-0.79)^{\mathbf{b}}$ & $0.46(0.35-0.62)^{\mathbf{a}}$ \\
\hline $36-47$ & $0.12(0.07-0.19)^{\mathbf{a}}$ & $0.30(0.20-0.46)^{a}$ & $0.31(0.20-0.49)^{\mathbf{a}}$ & $0.31(0.23-0.42)^{\mathbf{a}}$ \\
\hline $48-59$ & $0.09(0.06-0.15)^{\mathbf{a}}$ & $0.22(0.14-0.33)^{a}$ & $0.26(0.16-0.43)^{\mathbf{a}}$ & $0.25(0.18-0.34)^{\mathbf{a}}$ \\
\hline \multicolumn{5}{|l|}{ Recent episodes of fever } \\
\hline No & 1.00 & 1.00 & 1.00 & 1.00 \\
\hline Yes & $1.57(1.29-1.90)^{\mathbf{a}}$ & $1.68(1.19-2.37)^{\mathbf{a}}$ & $1.25(0.93-1.68)$ & $1.09(0.84-1.43)$ \\
\hline \multicolumn{5}{|l|}{ Recent episodes of diarrhea } \\
\hline No & 1.00 & 1.00 & 1.00 & 1.00 \\
\hline Yes & $0.95(0.73-1.24)$ & $1.07(0.74-1.55)$ & $1.21(0.86-1.70)$ & $1.07(0.84-1.35)$ \\
\hline \multicolumn{5}{|l|}{ Children are stunted } \\
\hline No & 1.00 & 1.00 & 1.00 & 1.00 \\
\hline Yes & $1.33(1.10-1.61)^{\mathbf{b}}$ & $1.49(1.17-1.89)^{\mathbf{a}}$ & $1.50(1.05-2.15)^{\mathbf{c}}$ & $1.17(0.97-1.42)$ \\
\hline \multicolumn{5}{|l|}{ Children are underweight } \\
\hline No & 1.00 & 1.00 & 1.00 & 1.00 \\
\hline Yes & $1.22(0.91-1.62)$ & $1.19(0.84-1.69)$ & $0.65(0.42-1.02)$ & $1.26(0.94-1.69)$ \\
\hline \multicolumn{5}{|l|}{ Mother's age in years } \\
\hline $15-24$ & 1.00 & 1.00 & 1.00 & 1.00 \\
\hline $25-34$ & $0.92(0.75-1.14)$ & $0.90(0.69-1.16)$ & $0.70(0.51-0.95)^{\mathbf{c}}$ & $0.89(0.74-1.08)$ \\
\hline $35-49$ & $0.96(0.74-1.25)$ & $0.92(0.68-1.24)$ & $0.66(0.46-0.95)^{c}$ & $0.83(0.65-1.06)$ \\
\hline \multicolumn{5}{|l|}{ Mother's educational level } \\
\hline No education & 1.00 & 1.00 & 1.00 & NA \\
\hline Primary & $0.87(0.66-1.13)$ & $1.09(0.85-1.40)$ & $0.82(0.46-1.45)$ & NA \\
\hline Secondary and above & $0.73(0.50-1.06)$ & $0.66(0.42-1.04)$ & $0.83(0.47-1.44)$ & NA \\
\hline \multicolumn{5}{|l|}{ Household wealth index } \\
\hline Poorest & 1.00 & 1.00 & 1.00 & 1.00 \\
\hline Poor & $0.88(0.50-1.06)$ & $0.89(0.64-1.25)$ & $1.53(1.01-2.30)^{c}$ & $0.96(0.75-1.22)$ \\
\hline Middle & $0.96(0.73-1.27)$ & $0.80(0.56-1.13)$ & $1.09(0.72-1.66)$ & $0.82(0.64-1.06)$ \\
\hline Richer & $0.81(0.60-1.08)$ & $0.84(0.56-1.27)$ & $1.08(0.62-1.88)$ & $0.91(0.67-1.23)$ \\
\hline
\end{tabular}


Table 3 Measures of association and variation between individual- and community-level factors and childhood anemia (Continued)

\begin{tabular}{|c|c|c|c|c|}
\hline Characteristic & Malawi & Mozambique & Namibia & Zimbabwe \\
\hline Richest & $0.69(0.48-0.99)^{\mathbf{c}}$ & $0.68(0.38-1.24)$ & $0.74(0.36-1.53)$ & $0.76(0.53-1.11)$ \\
\hline \multicolumn{5}{|l|}{ Community-level (clusters) } \\
\hline \multicolumn{5}{|c|}{ Community maternal anemia ${ }^{\dagger}$} \\
\hline Low anemia & 1.00 & 1.00 & 1.00 & 1.00 \\
\hline High anemia & $1.19(0.93-1.54)$ & $1.52(1.19-1.94)^{\mathrm{a}}$ & $1.478(0.97-2.25)$ & $0.778(0.63-1.03)$ \\
\hline \multicolumn{5}{|l|}{ Place of residence } \\
\hline Urban & 1.00 & 1.00 & 1.00 & 1.00 \\
\hline Rural & $1.07(0.71-1.60)$ & $1.14(0.80-1.63)$ & $0.87(0.61-1.25)$ & $0.78(0.58-1.03)$ \\
\hline \multicolumn{5}{|l|}{ Community wealth $^{\dagger+}$} \\
\hline Low & 1.00 & 1.00 & 1.00 & NA \\
\hline High & $0.91(0.69-1.19)$ & $0.78(0.53-1.14)$ & $0.84(0.53-1.34)$ & NA \\
\hline \multicolumn{5}{|c|}{ Community female education ${ }^{\S}$} \\
\hline Low & 1.00 & 1.00 & 1.00 & NA \\
\hline High & $0.82(0.65-1.03)$ & $1.14(0.83-1.55)$ & $0.801(0.50-1.29)$ & NA \\
\hline \multicolumn{5}{|c|}{ Community distance to $\mathrm{HF}^{¥}$} \\
\hline Low & 1.00 & 1.00 & 1.00 & NA \\
\hline High & $1.33(1.07-1.65)^{\mathbf{b}}$ & $0.98(0.73-1.33)$ & $0.99(0.72-1.36)$ & NA \\
\hline \multicolumn{5}{|c|}{ Community safe water access $^{\ddagger}$} \\
\hline Low & 1.00 & 1.00 & NA & 1.00 \\
\hline High & $0.90(0.72-1.12)$ & $0.78(0.57-1.07)$ & NA & $0.91(0.73-1.14)$ \\
\hline \multicolumn{5}{|c|}{ Measures of variation or clustering } \\
\hline \multicolumn{5}{|l|}{ Community level } \\
\hline$[\mathrm{T}(\mathrm{SE})]^{9}$ & $0.193(0.084)^{c}$ & $0.418(0.120)^{\mathbf{a}}$ & $0.157(0.126)$ & $0.086(0.048)^{c}$ \\
\hline$[\mathrm{T}(\mathrm{SE})]$ & $0.056(0.080)$ & $0.280(0.114)^{\mathbf{b}}$ & $0.070(0.129)$ & $0.064(0.050)$ \\
\hline$M O R^{ð}$ & 1.52 & 1.85 & 1.46 & 1.32 \\
\hline MOR & 1.25 & 1.66 & 1.29 & 1.27 \\
\hline ICC (\%) & 5.54 & 11.27 & 4.55 & 2.55 \\
\hline ICC (\%) & 1.68 & 7.84 & 2.09 & 1.90 \\
\hline PCV $\quad(\%)$ & 70.94 & 32.98 & 55.31 & 26.07 \\
\hline \multicolumn{5}{|l|}{ Model fit statistics } \\
\hline DIC (-2log likelihood) & 2935.72 & 2220.25 & 1397.00 & 3282.89 \\
\hline
\end{tabular}

Notes: Note: ${ }^{\mathrm{a}} \boldsymbol{P}<0.0001 ;{ }^{\mathbf{b}} \boldsymbol{P}<0.001 ;{ }^{c} \boldsymbol{P}<0.05 ; \neq$ aOR, adjusted odds ratio; $\mathrm{Cl}$, confidence interval; [T (SE)], community-level variance; $\mathrm{SE}$, standard error; MOR, median odds ration; ICC, intraclass correlation; PCV, proportional change in variance; the proportional change in variance expresses the change in the community level variance between the null model and the individual level model, and between the individual level model, and the model further including the community level covariate; DIC, deviation information criterion. Model I: unconditional model with random intercepts and had no predictors. Model II: contained a random-intercept fixed-slope and adjusted for individual-level factors. Model III: contained a random-intercept fixed-slope and adjusted for community contextual factors. Model IV: contained a random-intercept fixed-slope and controlled for both individual and community-level factors. The Bold texts indicate a statistically significant association at a $p$-value less than $0.05 .{ }^{\text {J }}$ percent of women with fertility rate of 5 children and above; ${ }^{\text {t+ }}$ percent of households categorized above $60 \%$ of wealth index; ${ }^{5}$ percent of women with primary school education and above; ${ }^{*}$ percent of households perceived distance to the nearest health facility as a big problem; ${ }^{*}$ percent of household with access to clean and safe drinking water sources specified by WHO/Unicef [34]; HF, health facility. ${ }^{\circ}$ Median Odds Ratio for null Model; IInterclass Correlation for null Model; ${ }^{\circ}$ community-level variance for null Model; NA variable had $p$-value greater than 0.25 at chi-square

of the variance in childhood anemia across communities in Malawi, Mozambique, Namibia, and Zimbabwe respectively were explained by, individual- and community-level. The MOR showed that the odds of childhood anemia increased by about 25, 84, 9 and 90\% in Malawi, Mozambique, Namibia, and Zimbabwe respectively when a mother moved from low to the high-risk community.

\section{Discussion}

Anemia reduces oxygen transport in the body, resulting in potentially permanent growth and developmental consequences for pre-school children [1, 3-5]. In this study of four Southern African countries, maternal anemia was highly associated with childhood anemia in all the four countries after controlling for the individual 
and community level factors. In addition, the results have also demonstrated that community-level maternal anemia exhibits a strong association with childhood anemia in Mozambique.

Individual-level factors showed that with an increase in infant's age the risk of childhood anemia decreases in all the four countries. The presence of fever two weeks prior to data collection and history of stunting (in Malawi Mozambique and Namibia), residing in poorest households (for Malawi) appeared to increase the risk of childhood anemia. For community-level factors, communities with a high percentage of women perceiving distance to the health facility as a big problem exacerbated adverse effects of maternal anemia on childhood anemia.

Our study has added a new knowledge that maternal anemia and communities with a high percentage of anemic women have adverse effects on childhood anemia beyond the pregnancy. The possible explanation is that mothers with anemia could be residing in poor households or poor communities which are indicators of socioeconomic deprivation. Thus, mothers might have problems in purchasing and providing good nutritious food for themselves as well as their children which might result in anemia due to inadequate intake of iron and other micronutrient-rich foods [36]. This is true because, within 12 months after birth, mothers and their children share a similar sociological environment, thus, their dietary patterns and quality of life may be similar [37]. Also, mothers and their children may share similar exposure to infections such as helminthiasis, malaria and other infectious diseases that may interfere with their red blood cells production and iron stores [38]. Additionally, low levels of essential minerals such as iron, zinc and folate as well as vitamin A and B12 in the breast milk of the anemic mother could also affect the $\mathrm{Hb}$ level of the breastfeeding child [39].

In addition, we found out that younger children were more likely to be anemic. This result is consistent with previous findings [10, 18, 40,41]. During the infancy-childhood growth spurt (6-12 months), children are particularly venerable to anemia due to the increase growth rate and subsequent demand for nutrients. Therefore an inadequate intake of exogenous iron during this period could lead to anemia [40]. In addition, infection resulting from the ingestion of impure foods and water [42, 43] could lead to symptoms such as diarrhea, vomiting and mouth ulcers which further affect their ability to ingest and absorb iron and other micronutrients $[43,44]$.

As reported in previous studies [20,36], we found that the presence of fever in the previous two weeks and stunting were positively associated with childhood anemia. Anemia can result from infections such as malaria and helminthiasis that are associated with fever [45].
Malaria and helminthiasis have also been implicated in increase RBC destruction with accompanying decreased replacement by the bone marrow [2, 46, 47]. Moreover, anemia is exacerbated by underlying inflammation, with subsequent iron imbalance and decreased erythrocyte level [48]. Previous reports have also implicated several pro-inflammatory cytokines in chronic anemia [49].

Furthermore, stunting denotes chronic food shortage, long-term effects of low iron intake and other micronutrient deficiencies, impaired immunity which is associated with low concentrations of $\mathrm{Hb}$ in the socioeconomically disadvantaged household [50]. In this study, living in poor and poorest households exhibited negative effects on childhood anemia in Malawi and Namibia.

Our findings also show that community-level factors increased the adverse effects of maternal anemia on childhood anemia in the Southern African countries. Communities with a high percentage of women perceiving distance to the health facility as a big problem increased the odds of childhood anemia. Distance to the nearest health facility is broadly linked to poor child health outcomes since access to health services are low. For instance, malaria and helminth infections are most common ailments in under 5 children in Sub-Saharan Africa and other developing counties, and anemia could be a complication of these diseases if not timely and effectively treated [51, 52]. In addition, unsafe drinking water supply may increase episodes of gastrointestinal infections which in turn are greatly linked to anemia due to depletion of iron stores and hemolysis of RBCs [46].

Our study is not without limitations. The cross-sectional nature of the data could not allow us to infer causality. The analysis was limited to children whose households were selected for $\mathrm{Hb}$ estimation.

The HemoCue system was used for measuring $\mathrm{Hb}$ levels to determine anemia. Additional studies may require using other $\mathrm{Hb}$ indices. Finally, we cannot exclude the effects of recall and social desirability bias on self-reported data.

\section{Conclusion}

Our study results indicated that maternal anemia was significantly associated with childhood anemia in all four countries. In addition, the individual- and community-level factors increased the adverse effects of maternal anemia on childhood anemia in Southern Africa countries. Public health policy and interventions aimed at reducing childhood anemia should focus on maternal anemia with emphasis on addressing iron and other micronutrient deficiencies, infections and health care practices of mothers with children below the age of 35 months. Distance to the nearest health facility will also need to be addressed at the community level. 


\section{Additional file}

Additional file 1: VIF and Tolerance for the four selected Southern African countries. Table S1 shows the multicollinearity using VIF and Tolerance for the four selected Southern African countries. (DOC 72 kb)

\begin{abstract}
Abbreviations
2SD: Two standard deviations; aOR: Adjusted odds ratio; CDC: Center for Disease Control; Cl: Confidence interval; DHS: Demographic and Health survey; DIC: Deviation information criterion; GLMM: Generalized linear mixed model; Hb: Hemoglobin; Hct: Hematocrit; HIV: Human immunodeficiency virus; ICCs: Intra-class correlations; IFC: International Finance Corporation; IL: Interleukin; IRB: Institutional Review Board; MMLR: Multilevel multivariable logistic regression; PCV: Percentage change in variation; PSU: Primary sampling unit; RBC: Red blood cell; SSA: Sub-Saharan Africa; TNF: Tumor necrosis factor; UNICEF: United Nations International Children's Education Fund; VIF: Variance inflation factor; WHO: World Health Organization
\end{abstract}

\section{Acknowledgments}

The authors are sincerely grateful to the MEASURE DHS for providing us with the population-based dataset through their archives.

\section{Availability of data and materials}

The datasets generated and/or analyzed during the current study are available in the DHS Program repository, http://dhsprogram.com/data/ available-datasets.cfm.

\section{Authors' contributions}

All authors contributed to the conception and design of the study. PAMN acquired the data, ON and TS conducted analysis and interpretation of the results. PAMN and PB drafted the first article. ON and TS revised the draft critically for important intellectual content. All authors reviewed and approved the final version of the manuscript

\section{Ethics approval and consent to participate}

This study was conducted according to the guidelines laid down in the Declaration of Helsinki and all procedures involving human subjects were approved by the Malawi National Health Science Research Committee, National Bioethics Commission of Mozambique (Comissao de Bioetica Nacional), Namibia Ministry of Health and Social Services, Medical Research Council of Zimbabwe and the Institutional Review Board (IRB) of ICF Macro, and the Centers for Disease Control (CDC) in Atlanta. Informed consent was obtained at the beginning of each interview and the authors sought permission from the DHS program for the use of the data.

\section{Competing interests}

The authors declare that they have no competing interests.

\section{Publisher's Note}

Springer Nature remains neutral with regard to jurisdictional claims in published maps and institutional affiliations.

\section{Received: 9 January 2018 Accepted: 15 May 2018}

Published online: 22 May 2018

\section{References}

1. Gener JPAD, Glader BE, Paraskevas F, Foerster J, Lukens JN, et al. Wintrobe's clinical hematology. 13th ed. Philadelphia: Lippincott Williams \& Wilkins; 2013. p. 2312.

2. Kai OK, Robert DJ. The pathophysiology of malarial anaemia: where have all the red cells gone? BMC Med. 2008;6:4.

3. $\mathrm{WHO}$. Haemoglobin concentrations for the diagnosis of anaemia and assessment of severity. Vitamin and Mineral Nutrition Information System. Geneva: WHO; 2011: Available from: http://www.who.int/vmnis/indicators/ haemoglobin.pdf. Accessed 10 Oct 2017.

4. Soliman AT, De Sanctis V, Kalra S. Anemia and growth. Indian J Endocrinol Metab. 2014;18(Suppl 1):S1-5.

5. Wirth JP, Rohner F, Woodruff BA, Chiwile F, Yankson H, Koroma AS, Russel F, Sesay F, Dominguez E, Petry N, et al. Anemia, micronutrient deficiencies, and malaria in children and women in Sierra Leone prior to the Ebola outbreak - findings of a cross-sectional study. PLoS One. 2016;11(5): e0155031.

6. Gutema B, Adissu W, Asress Y, Gedefaw L. Anemia and associated factors among school-age children in Filtu town, Somali region, Southeast Ethiopia. BMC hematology. 2014;14(1):13.

7. WHO. The global prevalence of anaemia in 2011. Geneva: World Health Organization; 2015.

8. Ray SCJ, Bhattacharjee J, Sharma S, Agarwala A. Determinants of nutritional anaemia in children less than five years age. IJCP. 2016;3:5.

9. Scott SP, Chen-Edinboro LP, Caulfield LE, Murray-Kolb LE. The impact of anemia on child mortality: an updated review. Nutrients. 2014;6(12):5915-32.

10. Ngesa $\mathrm{O}, \mathrm{Mwambi} H$. Prevalence and risk factors of anaemia among children aged between 6 months and 14 years in Kenya. PLoS One. 2014; 9(11):e113756.

11. Simbauranga RH, Kamugisha E, Hokororo A, Kidenya BR, Makani J. Prevalence and factors associated with severe anaemia amongst under-five children hospitalized at Bugando medical Centre, Mwanza, Tanzania. BMC hematology. 2015;15:13.

12. Magalhaes RJ, Clements AC. Mapping the risk of anaemia in preschool-age children: the contribution of malnutrition, malaria, and helminth infections in West Africa. PLoS Med. 2011;8(6):e1000438.

13. Crawley J. Reducing the burden of anemia in infants and young children in malaria-endemic countries of Africa: from evidence to action. Am J Trop Med Hyg. 2004;71(2 Suppl):25-34.

14. Semba RD, Bloem MW. The anemia of vitamin a deficiency: epidemiology and pathogenesis. Eur J Clin Nutr. 2002;56(4):271-81.

15. Calis JC, Phiri KS, Faragher EB, Brabin BJ, Bates I, Cuevas LE, de Haan RJ, Phiri Al, Malange $\mathrm{P}$, Khoka M, et al. Severe anemia in Malawian children. Malawi Med J. 2016;28(3):99-107.

16. Williams TN, Uyoga S, Macharia A, Ndila C, McAuley CF, Opi DH, Mwarumba S, Makani J, Komba A, Ndiritu MN, et al. Bacteraemia in Kenyan children with sickle-cell anaemia: a retrospective cohort and case-control study. Lancet. 2009;374(9698):1364-70.

17. Morris CR, Singer ST, Walters MC. Clinical hemoglobinopathies: iron, lungs and new blood. Curr Opin Hematol. 2006;13(6):407-18.

18. Zuffo CRK, Osório MM, Taconeli CA, Schmidt ST, da Silva BHC, Almeida CCB. Prevalence and risk factors of anemia in children. J Pediatr. 2016; 92(4):353-60.

19. Cardoso MA, Scopel KK, Muniz PT, Villamor E, Ferreira MU. Underlying factors associated with anemia in Amazonian children: a population-based, cross-sectional study. PLoS One. 2012;7(5):e36341.

20. Ngnie-Teta I, Receveur O, Kuate-Defo B. Risk factors for moderate to severe anemia among children in Benin and Mali: insights from a multilevel analysis. Food Nutr Bull. 2007;28(1):76-89.

21. Guidotti RJ. Anaemia in pregnancy in developing countries. BJOG. 2000; 107(4):437-8.

22. Rahman MM, Abe SK, Rahman MS, Kanda M, Narita S, Bilano V, Ota E, Gilmour S, Shibuya K. Maternal anemia and risk of adverse birth and health outcomes in low- and middle-income countries: systematic review and meta-analysis. Am J Clin Nutr. 2016;103(2):495-504.

23. Haider BA, Olofin I, Wang M, Spiegelman D, Ezzati M, Fawzi WW. Anaemia, prenatal iron use, and risk of adverse pregnancy outcomes: systematic review and meta-analysis. BMJ (Clinical Res ed). 2013;f3443:346.

24. Akhter S, Momen MA, Rahman MM, Parveen T, Karim RK. Effect of maternal anemia on fetal outcome. Mymensingh Med J. 2010;19(3):391-8.

25. The DHS Program > What-We-Do > Methodology. Available from: http:// dhsprogram.com/What-We-Do/Survey-Types/DHS-Methodology.cfm (2010). Acessed 21 Oct 2017.

26. Be WK, Kerkkamp HE, Booij LH. Hemocue-a new haemoglobinometer in the clinic. Eur J Anaesthesiol. 1991;8(1):55-8.

27. Shamah Levy T, Mendez-Gomez-Humaran I, Morales Ruan MD, Martinez Tapia B, Villalpando Hernandez S, Hernandez Avila M. Validation of Masimo pronto 7 and HemoCue 201 for hemoglobin determination in children from 1 to 5 years of age. PLoS One. 2017;12(2):e0170990.

28. WHO/UNICEF: Why children are still dying and what can be done; 2009

29. WHO Child Growth Standards based on length/height, weight and age. Acta paediatr (Oslo, Norway: 1992) Suppl. 2006;450:76-85.

30. Machisa M, Wichmann J, Nyasulu PS. Biomass fuel use for household cooking in Swaziland: is there an association with anaemia and stunting in children aged 6-36 months? Trans R Soc Trop Med Hyg. 2013;107(9):535-44. 
31. Kyu HH, Georgiades K, Boyle MH. Biofuel smoke and child anemia in 29 developing countries: a multilevel analysis. Ann Epidemiol. 2010;20(11): $811-7$.

32. RSa KJ. The DHS Wealth Index. Calverton: DHS; 2004.

33. Diez Roux AV. A glossary for multilevel analysis. J Epidemiol Community Health. 2002;56(8):588-94.

34. WHO/UNICEF. WHO and UNICEF Joint Monitoring Programme (JMP) for water supply and sanitation: types of drinking-water sources and sanitation, Geneva WHO/UNICEF. Available from: http://www.unwater.org/publication_ categories/whounicef-joint-monitoring-programme-for-water-supplysanitation-hygiene-jmp/ (2016). Acessed 28 Oct 2017.

35. Maldonado G, Greenland S. Simulation study of confounder-selection strategies. Am J Epidemiol. 1993;138(11):923-36.

36. Ngwira A, Kazembe LN. Bayesian random effects modelling with application to childhood anaemia in Malawi. BMC Public Health. 2015:15:161.

37. Gao W, Yan H, Wang D, Dang S, Pei L. Severity of anemia among children under 36 months old in rural western China. PLoS One. 2013;8(4):e62883.

38. Brooker S, Akhwale W, Pullan R, Estambale B, Clarke SE, Snow RW, Hotez PJ. Epidemiology of plasmodium-helminth co-infection in Africa: populations at risk, potential impact on anemia, and prospects for combining control. Am J Trop Med Hyg. 2007;77(6 Suppl):88-98.

39. Wang J, Wang H, Chang S, Zhao L, Fu P, Yu W, Man Q, Scherpbier R, Pan L, Duan $Y$, et al. The influence of malnutrition and micronutrient status on anemic risk in children under 3 years old in poor areas in China. PLoS One. 2015;10(10):e0140840.

40. Zhao A, Zhang Y, Peng Y, Li J, Yang T, Liu Z, Lv Y, Wang P. Prevalence of anemia and its risk factors among children 6-36 months old in Burma. Am J Trop Med Hyg. 2012;87(2):306-11.

41. Austin AM, Fawzi W, Hill AG. Anaemia among Egyptian children between 2000 and 2005: trends and predictors. Matern Child Nutr. 2012;8(4):522-32.

42. Kamal MM, Hasan MM, Davey R. Determinants of childhood morbidity in Bangladesh: evidence from the demographic and health survey 2011. BMJ Open. 2015;5(10):e007538.

43. Woldie H, Kebede $\mathrm{Y}$, Tariku A. Factors associated with Anemia among children aged 6-23 months attending growth monitoring at Tsitsika health center, wag-Himra zone, Northeast Ethiopia. J Nutr Metab. 2015;2015: 928632.

44. Osorio MM, Lira Pl, Batista-Filho M, Ashworth A. Prevalence of anemia in children 6-59 months old in the state of Pernambuco, Brazil. Rev Panam Salud Publica. 2001;10(2):101-7.

45. Hotez PJ, Bundy DAP, Beegle K, Brooker S, Drake L, de Silva N, Montresor A, Engels D, Jukes M, Chitsulo L et al: Helminth infections: soil-transmitted helminth infections and schistosomiasis. In: Disease Control Priorities in Developing Countries. Edn. Edited by nd, Jamison DT, Breman JG, Measham AR, Alleyne G, Claeson M, Evans DB, Jha P, Mills A, Musgrove P. Washington (DC): The International Bank for Reconstruction and Development/the World Bank Group; 2006

46. Osazuwa F, Ayo OM, Imade P. A significant association between intestinal helminth infection and anaemia burden in children in rural communities of Edo state, Nigeria. N Am J Med Sci. 2011;3(1):30-4.

47. Koukounari A, Estambale BB, Njagi JK, Cundill B, Ajanga A, Crudder C, Otido J, Jukes MC, Clarke SE, Brooker S. Relationships between anaemia and parasitic infections in Kenyan schoolchildren: a Bayesian hierarchical modelling approach. Int J Parasitol. 2008;38(14):1663-71.

48. Weiss G, Goodnough LT. Anemia of chronic disease. N Engl J Med. 2005; 352(10):1011-23.

49. Jiang B, Snipes-Magaldi L, Dennehy P, Keyserling H, Holman RC, Bresee J, Gentsch J, Glass RI. Cytokines as mediators for or effectors against rotavirus disease in children. Clin Diagn Lab Immunol. 2003;10(6):995-1001.

50. Khan JR, Awan N, Misu F. Determinants of anemia among 6-59 months aged children in Bangladesh: evidence from nationally representative data. BMC Pediatr. 2016;16:3.

51. Njua-Yafi C, Achidi EA, Anchang-Kimbi JK, Apinjoh TO, Mugri RN, Chi HF, Tata RB, Njumkeng C, Nkock EN, Nkuo-Akenji T. Malaria, helminths, coinfection and anaemia in a cohort of children from Mutengene, south western Cameroon. Malar J. 2016;15:69.

52. Kinung'hi SM, Magnussen P, Kaatano GM, Kishamawe C, Vennervald BJ. Malaria and helminth co-infections in school and preschool children: a cross-sectional study in Magu district, North-Western Tanzania. PLoS One. 2014;9(1):e86510.

Ready to submit your research? Choose BMC and benefit from:

- fast, convenient online submission

- thorough peer review by experienced researchers in your field

- rapid publication on acceptance

- support for research data, including large and complex data types

- gold Open Access which fosters wider collaboration and increased citations

- maximum visibility for your research: over $100 \mathrm{M}$ website views per year

At BMC, research is always in progress.

Learn more biomedcentral.com/submissions 